\title{
Characterisation of Major Fault Detection Features and Techniques for the Condition-Based Monitoring of High-speed Centrifugal Blowers
}

\author{
Samer Gowid \\ Department of Electronic, Electrical and systems Engineering, Faculty of Engineering, Loughborough University, \\ $U K$ \\ Department of Mechanical Engineering and Industrial Engineering, College of Engineering, Qatar University, \\ Qatar
}

\section{Roger Dixon}

Department of Electronic, Electrical and systems Engineering, Faculty of Engineering, Loughborough University, $U K$

\author{
Saud Ghani \\ Department of Mechanical Engineering and Industrial Engineering, College of Engineering, Qatar University, \\ Qatar
}

(Received 25 March 2014; accepted 30 September 2015)

\begin{abstract}
This paper investigates and characterises the major fault detection signal features and techniques for the diagnostics of rotating element bearings and air leakage faults in high-speed centrifugal blowers. The investigation is based on time domain and frequency domain analysis, as well as on process information, vibration, and acoustic emission fault detection techniques. The results showed that the data analysis method applied in this study is effective, as it yielded a detection accuracy of $100 \%$. A lookup table was compiled to provide an integrated solution for the developer of Condition-Based Monitoring (CBM) applications of centrifugal blowers. The major contribution of this paper is the integration and characterisation of the major fault detection features and techniques.
\end{abstract}

\section{INTRODUCTION}

Condition-Based Monitoring (CBM) is a strategy aimed at extending machine life, lowering maintenance cost, and increasing both productivity and profitability. ${ }^{1}$ Unlike preventative maintenance, which is based on servicing a machine at scheduled intervals, CBM relies upon actual machine health condition to diagnose faults and to determine when the maintenance is required. The specific advantage of condition monitoring is that potential degradation or failure can be detected. This technique enables the user to take maximum advantage of the useful life of a component, such as a bearing, since the equipment can be left in service if its operational performance meets the desired performance standards. ${ }^{2}$

Centrifugal compressors are widely used in the industry, and in particular in the oil and gas industries, as they compress the propane and mixed refrigerants in the liquefaction process. A 15 HP industrial centrifugal blower was employed for the emulation of high-speed centrifugal blowers. Due to the similarity between centrifugal blowers and centrifugal compressors, ${ }^{3}$ this work can be extended to centrifugal compressors and centrifugal equipment.

The global structure of the generally used monitoring system can be divided into three main sections: The first phase is data collection, with data reports gathered in a digital form. The second phase is acquisition, which entails calculation of the statistical values and functions in time and frequency domain with integrated data reduction by fault and operational pattern. The more difficult third phase of automatic fault diagnostics is still under development and permanently adapted to the necessities of industrial applications, mainly dependent on the acting personnel at the monitoring system. ${ }^{4}$

Machine condition, machine faults, and on-going damage can be identified in operating machines by fault symptoms and signatures. Mechanical vibration, acoustic emission (AE), and process information are the three major fault detection techniques in addition to monitoring changes in process operating parameters, such as pressure, temperatures, and efficiency. Thus, this study will provide a characteristics investigation based on these major techniques, which should be included in any full capabilities condition-based maintenance system. Integrating these techniques can yield early detection and trending of numerous equipment faults. Moreover, it could have a potential to reduce false alarms due to noise and fault interference issues.

Vibrations of machines are the results of the dynamic forces due to moving parts and structures (for example, foundations), which are interlinked to the machine and its mechanical properties. All machine components generate specific vibration signatures which are then transmitted to the machine's structure. Vibration analysis detects repetitive motions of a surface on rotating or oscillating machines. The repetitive motions may be caused by unbalance, misalignment, resonance, electrical effects, rolling element bearing faults, or many other problems. The various vibration frequencies in a rotating ma- 
chine are directly related to the geometry and the operating speed of the machine. By knowing the relationship between the frequencies and the types of defects, vibration analysts can determine the cause and severity of faults or problem conditions. The history of the machine and the previous degradation pattern are important factors in determining the current and future operating condition of the machine. Frequency, displacement, velocity acceleration, and phase angle are the major five characteristics of rotating machine vibration. ${ }^{5}$

Unlike the mechanical vibration technique, the $\mathrm{AE}$ technique is less affected by noise and detects faults such as friction in bearing in their early stages. All rotating equipment produces frictional forces with high frequency ultrasonic signatures, which are often masked by ambient plant noise and low frequency vibrations. ${ }^{6}$ As the defect size increased, acoustic emission, root mean square, maximum amplitude, and kurtosis values increased; however, observations of corresponding parameters from vibration measurements were disappointing. ${ }^{7}$

For rotating machinery, the most commonly measured AE parameters for diagnostics are amplitude, RMS, energy, kurtosis, crest factor, counts, and events. Observations of the frequency spectrum, whilst informative for traditional nondestructive evaluation, were found to have a limited success in machinery monitoring. This is primarily due to the broad frequencies associated with the sources of generation of $\mathrm{AE}$ in rotating machinery. For example, the transient impulse associated with the breakage of contacting surface asperities experiencing relative motion will excite a broad frequency range. ${ }^{6}$

The process parameters such as pressure, temperature, vibration, and flow rate, and material samples such as oil and air are also used to monitor machine conditions. With these parameters and samples, condition-based maintenance obtains indications of system and equipment health, performance, and integrity, and provides information for scheduling timely correction. ${ }^{8}$

Tandon and Nakra investigated AE counts and peak amplitudes for an outer race defect using a resonant type transducer. It was concluded that AE counts increased with increasing load and rotational speed. However, it was observed that AE counts could only be used for defect detection when the defect was less than $250 \mu \mathrm{m}$ in diameter, though AE peak amplitude provided an indication of defects irrespective of the defect size. ${ }^{8}$ Rogers utilised the AE technique for monitoring slow rotating anti-friction slew bearings on cranes employed for gas production, and obtained encouraging results compared to vibration monitoring techniques. Rubbing of the crack faces, grinding of the metal fragments in the bearing, and impacts between the rolling elements and the damaged parts in the loaded zone were identified as sources of detectable AE signatures. The author stated that "because of the slow rotational speed of the crane, application of conventional vibration analysis $(0-20 \mathrm{kHz})$ was of limited value for on-line condition monitoring." AE resonant transducers between $100 \mathrm{kHz}$ and $300 \mathrm{kHz}$ were found to be informative for online monitoring of bearings using kurtosis at different frequency bands. ${ }^{9}$ Wang and Hu investigated uncertainties and ambiguities that exist between pump fault symptoms and the events using a spectral features-based technique. The research resulted in an effective approach to solve the problem of fault diagnostics. Fuzzy logic was used to model the uncertainty and ambiguity relationship among different faults, analyse the fuzzy information that existed in the

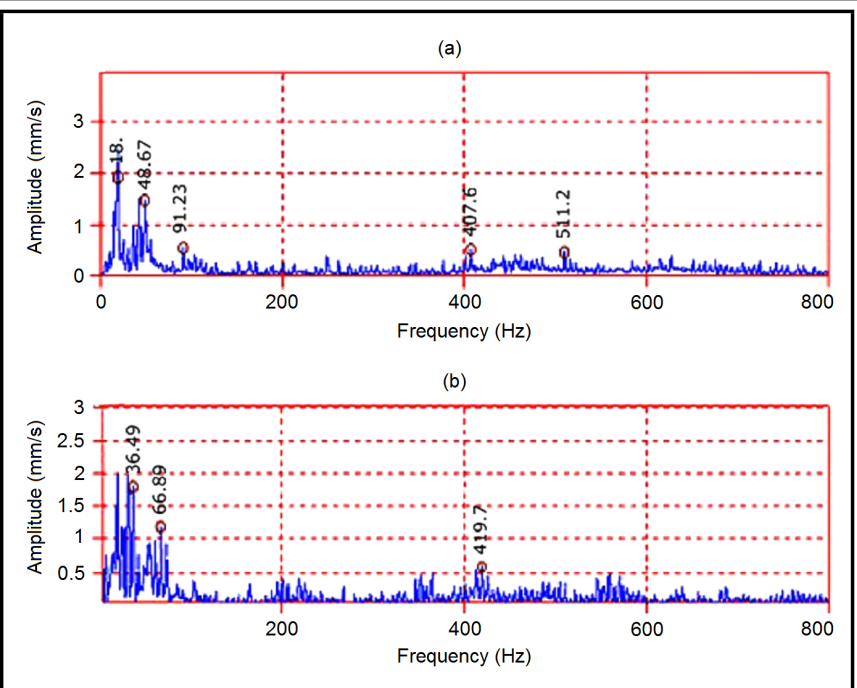

Figure 1. Two frequency spectra represent (a) sample fault, (b) second fault with the same sample fault on the second inlet valve. ${ }^{10}$

different phases of fault diagnostics and condition monitoring of the pump, and classify frequency spectra that represented various pump faults. ${ }^{10}$ The author concluded that the condition recognition and fault diagnostics were detected through the fuzzy comprehensive discrimination according to the defuzzy diagnostic criteria. Two vibration spectra for the faulty device are shown in Fig. 1. Schultheis, et al. studied different techniques used in machine heath condition monitoring of motors. They compared the online versus periodic monitoring and proven versus effective techniques. The following techniques were found to be effective: ultrasound vibration, mechanical vibration, temperature, rod run out, and pressure velocity measurements. For gas leaks, ultrasonic vibration measurement was preferable to mechanical vibration. The online monitoring was effective in decreasing the chance of catastrophic failures, as well as maintenance and shutdown costs. ${ }^{11}$

Based on the above research, the AE, vibration, and process information are the most utilised CBM techniques. It can be also concluded that the acoustic emission technique proved its effectiveness over other techniques for $\mathrm{CBM}$ of rotating equipment. The utilisation of the multi-fault detection technique maximises the efficiency and accuracy of diagnosing faults. The fault detection technique must be properly selected based on the fault type.

This paper is divided into eight sections. The first section provides an introduction to CBM and fault detection techniques. Section two describes the methodology employed in this study. Section three illustrates the experimental setup, while section four shows the design of the experiment. Section five presents the results of the fault diagnostic using the major fault detection techniques. Section six presents the developed lookup table, which summarizes the results of the characteristics investigation. Section seven discusses the results, and section eight concludes the results of the study.

\section{METHODOLOGY}

Three major fault detection techniques, in addition to five time domain and frequency domain signal features, will be investigated and compared with respect to their capability of diagnosing a centrifugal air blower's faults using a $15 \mathrm{HP}$ in- 
dustrial air blower system, high-speed NI DAQ system, broad frequency range $\mathrm{AE}$ sensor, vibration sensor, and a pressure sensor.

This paper will utilise a recent Fast Fourier Transform (FFT)-based segmentation and features selection algorithm in the selection of best spectral feature sets. ${ }^{12}$ A "lookup table" will then be developed to characterise the major fault detection techniques and signal analysis methods for the condition-based monitoring of centrifugal blowers. The table will combine information from several fault detection techniques, including AE vibration, pressure, crest factor, energy factor, RMS, amplitude, and spectral features. This approach is found to have great potential for the development of CBM systems for typical centrifugal equipment and improves the accuracy of detection compared with the use of a single fault detection technique.

\section{EXPERIMENTAL SETUP}

Experimental tests were conducted in a laboratory environment hosted by Qatar University using a Paxton AT1200 industrial single-stage centrifugal air blower system. The blower has a maximum flow rate of 800 CFM @ 70" W/C. Figure 2 shows the single-stage centrifugal blower.

The air blower system consists of a 15 HP DC motor, a DC inverter for motor speed control, a 4" hose, a 4" air flow control valve, and a centrifugal air blower. Four factory calibrated AE sensors from Physical Acoustics were utilised to measure the acoustic signals, along with two low frequency range sensors with an operating range of 35-100 kHz (Model: R6a) and two high frequency range sensors with an operating range of 100$1000 \mathrm{kHz}$ (model: UT1000). The AE sensors were positioned as close as possible to the bearings, as shown in Figs. 3 and 4. However, the AE sensor can measure any frequency outside its operating bandwidth, but with less sensitivity. A $70 \mathrm{~g}$ triaxial vibration sensor was positioned midway between the shaft bearings, and a pressure sensor was installed in the outlet pipe and was positioned $50 \mathrm{~cm}$ away from the outlet of the blower.

The schematic of the experimental setup is shown in Fig. 4. The AE sensors were attached to signal conditioners and programmable low pass filters with isolated grounds to combat the problem of aliasing in sampling signals. A cut-off frequency of $200 \mathrm{kHz}$ was set to attenuate high frequency $\mathrm{AE}$ signals. The models of bearings A and B are DKT-7203BMP and FAG-2203TV, respectively. The data was collected using an MSeries- PCI 6250 National Instruments data acquisition board with 16 channels, 16-bit resolution, and a $1.25 \mathrm{MS} / \mathrm{s}$ sampling rate.

\section{DESIGN OF EXPERIMENT}

Bearing problems account for over $40 \%$ of machine breakdowns. ${ }^{8}$ Thus, this experimental work focuses on bearings faults in centrifugal blowers, and investigates the issue of fault interference, as well. Typical causes of bearing faults are excessive load, overheating, false brinelling, true brinelling, normal fatigue failure, reverse loading, contaminations, lubricant failure, corrosion, misalignment, loose fits, and tight fits. ${ }^{13}$ Two typical bearing failure modes were selected to evaluate the addressed detection techniques - true brinelling and normal fatigue failures. Brinelling occurs when loads exceed the

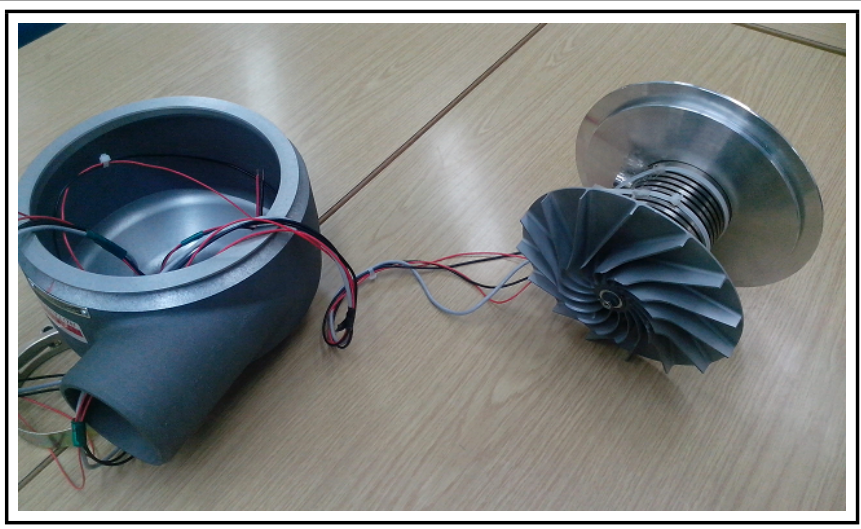

Figure 2. Single stage centrifugal blower.

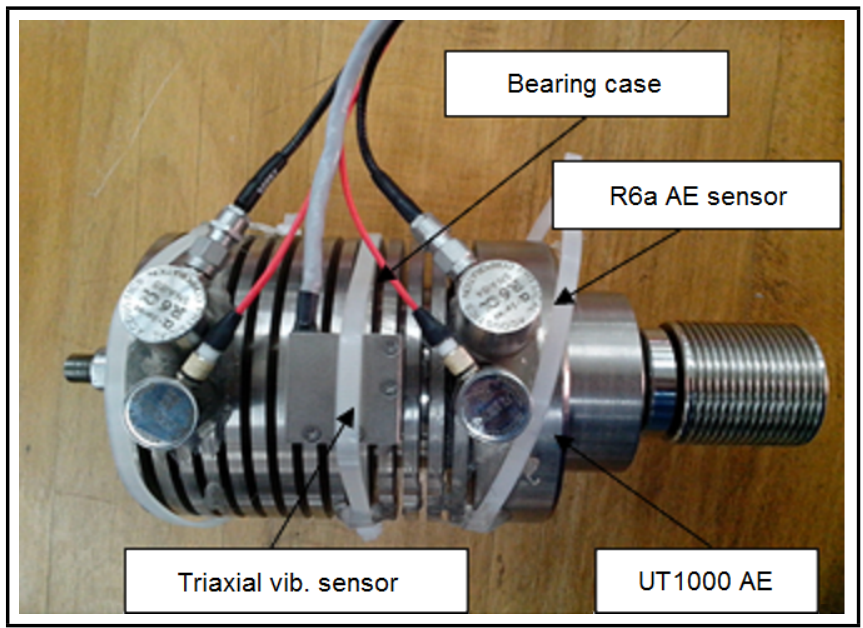

Figure 3. Positions of AE sensors.

elastic limit of the ring material. Brinell marks show as indentations in the raceways, and these increase bearing vibration (noise). Severe brinell marks can cause premature fatigue failure. Fatigue failure, usually referred to as spalling, is the fracture of the running surfaces and subsequent removal of small discrete particles of material. Spalling can occur on the inner ring, outer ring, or balls. ${ }^{13}$

Figure 5 illustrates the faults in bearings A and B. Bearing $\mathrm{A}$ has a $2 \mathrm{~mm}$ throughout hole in the outer race to emulate a brinelling fault, while bearing $\mathrm{B}$ has four notches in both sides with a maximum notch width of $1.5 \mathrm{~mm}$ to emulate a fatigue fault. Five Machine Conditions (MC) were emulated at an ambient temperature of $22^{\circ} \mathrm{C}$ as shown in Table 1 . Several tests were conducted under three different operational speeds to check the functionality and proper installation of sensors using the experimental setup shown in Fig. 3. To control the speed-related risks, the speed was increased from 3,600 to 6,960 RPM, and then to 15,650 RPM. The R6a sensor, which was directly positioned above bearing A, gave the highest reading at 15,650 RPM. Hence, as the experiment was designed to have only one AE sensor, the bearing A R6a sensor was selected for its proper installation and high sensitivity. In this study, the measured $\mathrm{AE}$ frequencies ranged from $2 \mathrm{kHz}$ to $121 \mathrm{kHz}$

Five experiments were conducted in a laboratory at the maximum blower rotational speed of 15,650 RPM (maximum power). The operating point of the blower was set to maximum to emulate industrial air blower systems. The first experiment 


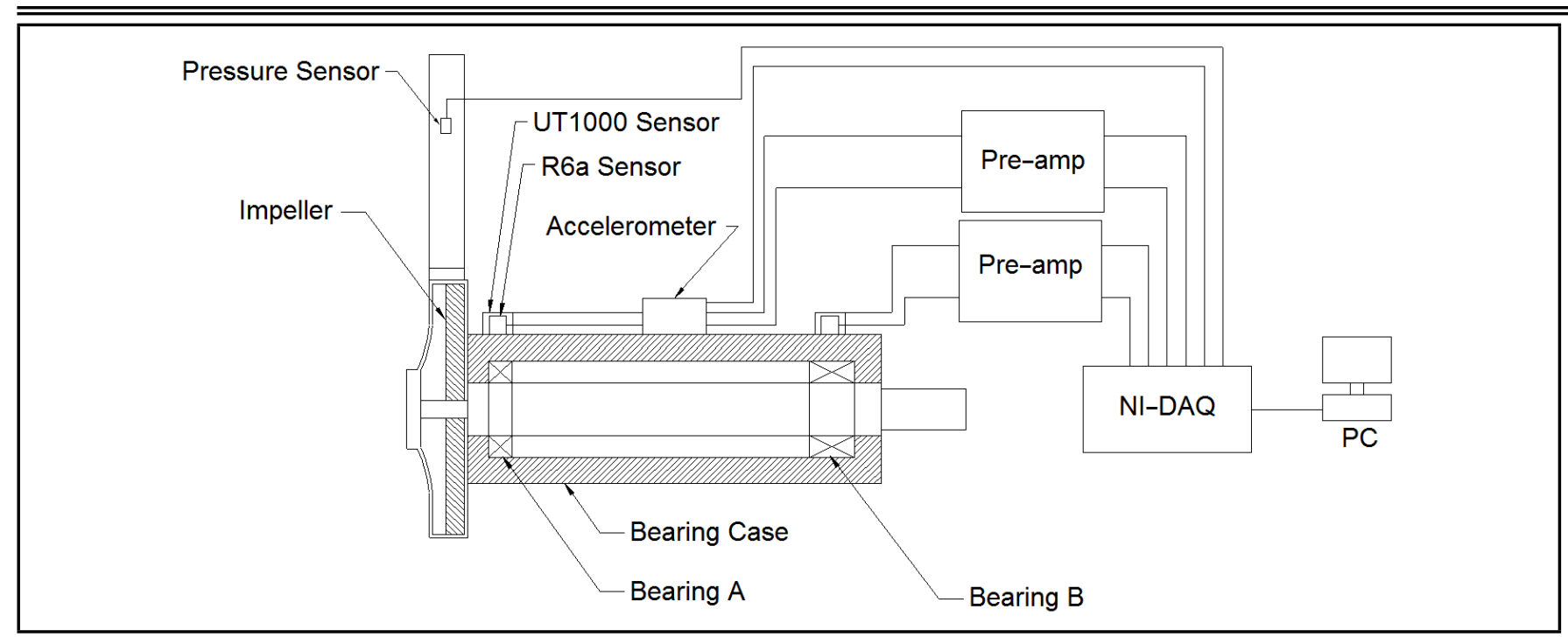

Figure 4. The schematic of the experimental setup.

Table 1. Machine health conditions.

\begin{tabular}{||c|c|c|c||}
\hline $\begin{array}{c}\text { Machine } \\
\text { Condition }\end{array}$ & Bearing A & Bearing B & Air leakage \\
\hline MC 1 & Healthy & Healthy & No \\
MC 2 & Healthy & Healthy & Yes \\
MC 3 & Outer race defect & Healthy & No \\
MC 4 & Healthy & Outer race defect & No \\
MC 5 & Outer race defect & Outer race defect & No \\
\hline
\end{tabular}

emulated the healthy condition, the second experiment emulated the air leakage problem, and the remaining experiments emulated the three bearing fault conditions. Faults emulated in MC 5 are a combination of MC 3 and MC 4 faults. The flow control valve was partially closed to maintain an outlet air pressure of 1.165 BarA. To emulate the air leakage problem (MC 2), the control valve was set to fully open. The majority of air leakages occur because of either a crack in blower case, rapture in hose, or a joint failure. The data were sampled using the high speed NI DAQ board at a sampling rate of $1 \mathrm{MS} / \mathrm{s}$ for a time period of 187 seconds. For each of the five conditions, 10 data sets were collected at a fixed time interval of 13 second (one set every 13 second). Each data set had a size of $1 \times 10^{6}$ samples and a sampling rate of $1 \mathrm{MHz}$. The first samples for the five machine conditions were taken 60 seconds after the blower reached its full rotation speed. Fifty percent of the 50 data set were used for training while the remaining sets were used for testing.

\section{FAULT DIAGNOSTIC USING MAJOR FAULT DETECTION TECHNIQUES}

In this section, the fault detection capabilities of the three major fault detection techniques ${ }^{14}$ are investigated and assessed for the diagnostics of typical centrifugal blowers' faults, namely Acoustic Emission (AE), vibration, and process information techniques.

\subsection{Acoustic Emission Technique}

The AE signals were measured using bearing A R6a AE sensor. Four samples were collected for each machine condition. Matlab was used to calculate the following time domain features: RMS, amplitude, crest factor, and energy. The frequency domain was also utilized and the AE spectral features

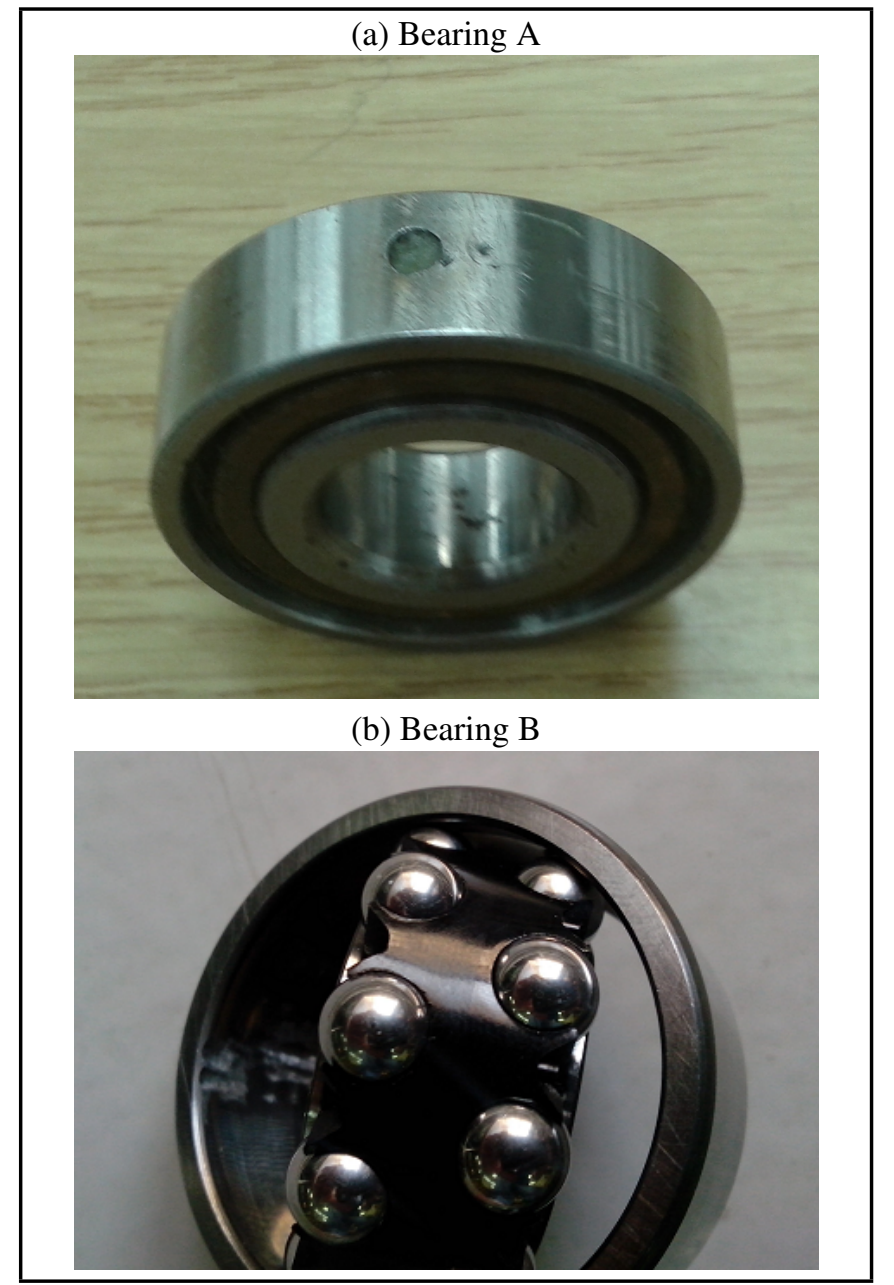

Figure 5. Notches in the outer races of bearings A and B.

were extracted. Machine conditions were grouped into three different groups. The first group includes MCs 1 and 2, the second group includes MCs 3 and 5, and the third group includes MC 4 only.

Table 2 shows the RMS values of training sets. The RMS values shown in Table 2 can be used to detect MC 1, MC 2, MC 4, and group 2. The principal drawback of the AE RMS feature is that it cannot be utilized for the detection of all ma- 


Table 2. AE RMS Values (V).
\begin{tabular}{||c|c|c|c|c|c|c|}
\hline & 1 & 2 & 3 & 4 & Min & Max \\
\hline MC 1 & 0.294 & 0.310 & 0.310 & 0.306 & 0.294 & 0.310 \\
MC 2 & 0.342 & 0.352 & 0.325 & 0.321 & 0.321 & 0.352 \\
MC 3 & 0.575 & 0.611 & 0.607 & 0.603 & 0.575 & 0.611 \\
MC 4 & 2.824 & 2.834 & 2.552 & 2.468 & 2.468 & 2.834 \\
MC 5 & 0.713 & 0.593 & 0.470 & 0.457 & 0.457 & 0.713 \\
\hline
\end{tabular}

Table 3. AE maximum amplitude values (V).

\begin{tabular}{||c|c|c|c|c|c|c||}
\hline & 1 & 2 & 3 & 4 & Min & Max \\
\hline MC 1 & 1.347 & 1.445 & 1.389 & 1.273 & 1.273 & 1.445 \\
MC 2 & 1.917 & 2.037 & 1.564 & 1.635 & 1.564 & 2.037 \\
MC 3 & 4.622 & 4.494 & 4.520 & 5.015 & 4.494 & 5.015 \\
MC 4 & 10.512 & 10.555 & 10.207 & 9.979 & 9.979 & 10.555 \\
MC 5 & 4.465 & 3.638 & 3.521 & 3.588 & 3.521 & 4.465 \\
\hline
\end{tabular}

Table 4. AE crest factors.

\begin{tabular}{|l|c|c|c|c|c|c|}
\hline & 1 & 2 & 3 & 4 & Min & Max \\
\hline MC 1 & 4.585 & 4.662 & 4.486 & 4.165 & 4.165 & 4.662 \\
MC 2 & 5.602 & 5.785 & 4.816 & 5.097 & 4.816 & 5.785 \\
MC 3 & 8.044 & 7.351 & 7.449 & 8.319 & 7.351 & 8.319 \\
MC 4 & 3.723 & 3.724 & 3.999 & 4.043 & 3.723 & 4.043 \\
MC 5 & 6.262 & 6.137 & 7.493 & 7.845 & 6.137 & 7.845 \\
\hline
\end{tabular}

chine conditions.

Table 3 shows the calculated signal maximum amplitudes of the training sets. The Maximum amplitude feature can be used to differentiate between all machine faults. For several machine conditions, the maximum amplitude values are close to each other, which will definitely affect the accuracy of detection.

Table 4 presents the calculated crest factors of training sets. The crest factor is equal to the RMS value divided by the maximum amplitude of the same signal. The crest factor feature can be utilized to differentiate between MC 1, MC 2, MC 4, and group 2. This time domain feature cannot be utilized for the detection of all machine faults.

Table 5 displays the AE energy values of training sets. The energy feature can be utilized to differentiate between MC 1, MC 2, MC 4, and group 2. As the difference between the energy values of MC 1 and MC 2 is large, this time series feature can be better utilized to differentiate between MC 1 and MC 2 which gives the energy feature a benefit over RMS, amplitude and crest factor features. The main drawback is that the energy feature cannot be utilized to detect all machine conditions.

Figure 6 illustrates the difference in the values of RMS, crest factor, amplitude, and energy time domain AE signal features. Although the energy feature is best in comparison to others, it cannot be fully utilized to differentiate between condition 3 and 4.

An FFT-based segmentation and features selection algorithm was utilized to check the suitability of AE spectral features for the detection of machine conditions. The range of the measured AE frequency was $2 \mathrm{kHz}$ to $121 \mathrm{kHz}$. Moreover, the algorithm investigated the segment sizes (number of divisions) that can be utilized for pattern classification. The selec-

Table 5. AE energy values (J).

\begin{tabular}{|l|c|c|c|c|c|c|}
\hline & 1 & 2 & 3 & 4 & Min & Max \\
\hline MC 1 & 86,261 & 96,088 & 95,869 & 93,358 & 86,261 & 96,088 \\
MC 2 & 117,079 & 124,004 & 105,439 & 102,940 & 102,940 & 124,004 \\
MC 3 & 330,158 & 373,780 & 368,167 & 363,459 & 330,158 & 373,780 \\
MC 4 & $7,973,092$ & $8,031,877$ & $6,514,243$ & $6,093,149$ & $6,093,149$ & $8,031,877$ \\
MC 5 & 508,308 & 351,403 & 220,829 & 209,173 & 209,173 & 508,308 \\
\hline
\end{tabular}


Figure 6. Graphical presentation for the AE RMS, amplitude, crest factor and energy values. 


Table 6. Segmented FFT AE spectra.
\begin{tabular}{|c|c|c|c|c|c|}
\hline S & MC 1 & MC 2 & MC 3 & MC 4 & MC 5 \\
\hline $121 \mathrm{kHz}$ & 0 & 1 & 0 & 1 & 0 \\
$120 \mathrm{kHz}$ & 0 & 1 & 0 & 1 & 0 \\
$119 \mathrm{kHz}$ & 1 & 1 & 1 & 1 & 1 \\
$118 \mathrm{kHz}$ & 0 & 0 & 1 & 1 & 1 \\
$117 \mathrm{kHz}$ & 1 & 1 & 0 & 1 & 1 \\
$116 \mathrm{kHz}$ & 1 & 1 & 0 & 1 & 1 \\
$115 \mathrm{kHz}$ & 0 & 1 & 0 & 1 & 1 \\
$114 \mathrm{kHz}$ & 0 & 1 & 1 & 1 & 1 \\
$113 \mathrm{kHz}$ & 0 & 1 & 1 & 1 & 1 \\
$112 \mathrm{kHz}$ & 0 & 1 & 1 & 1 & 1 \\
$111 \mathrm{kHz}$ & 0 & 1 & 1 & 1 & 1 \\
$110 \mathrm{kHz}$ & 0 & 1 & 1 & 1 & 1 \\
$109 \mathrm{kHz}$ & 0 & 1 & 1 & 1 & 1 \\
$108 \mathrm{kHz}$ & 1 & 1 & 1 & 1 & 1 \\
$107: 2 \mathrm{kHz}$ & 1 & 1 & 1 & 1 & 1 \\
$1 \mathrm{kHz}$ & 1 & 1 & 1 & 1 & 1 \\
\hline
\end{tabular}

Table 7. AE crest factors.

\begin{tabular}{|l|c|c|c|c|c|c|}
\hline & 1 & 2 & 3 & 4 & Min & Max \\
\hline MC 1 & 1.663 & 1.655 & 1.633 & 1.629 & 1.629 & 1.663 \\
MC 2 & 1.660 & 1.660 & 1.662 & 1.662 & 1.660 & 1.662 \\
MC 3 & 1.872 & 1.853 & 1.828 & 1.823 & 1.823 & 1.872 \\
MC 4 & 1.668 & 1.653 & 1.628 & 1.626 & 1.626 & 1.668 \\
MC 5 & 1.685 & 1.668 & 1.636 & 1.630 & 1.630 & 1.685 \\
\hline
\end{tabular}

tion of the most suitable maximum Segment size (S) depends on the detection accuracy required. ${ }^{12}$ Table 6 shows the detection accuracy at different segment sizes where 0 means that the fault cannot be detected at this maximum segment size, while 1 means that the fault can be detected. All machines conditions were detected at a maximum segment size of $108 \mathrm{kHz}$ and a confidence level of 3 . At this segment size, the AE frequency spectrum was divided into two divisions; the first frequency division ranged from $2 \mathrm{kHz}$ to $108 \mathrm{kHz}$, while the second division ranged from $108 \mathrm{kHz}$ to the maximum measured frequency, $121 \mathrm{kHz}$. At a segment size of $1 \mathrm{kHz}$, all machine conditions were successfully detected with a confidence level of 93. At this segment size, the AE frequency spectrum was divided into 119 equal divisions of $1 \mathrm{kHz}$ each.

The confidence level is defined as the difference between the highest number of matching features between the signal features and the corresponding fault benchmark features, and between the second highest number of matching features between the same signal features and another fault benchmark features. The larger the value of the difference, the better confidence level. ${ }^{12}$

\subsection{Vibration Technique}

Due to the high stiffness of the blower structure in the vertical and horizontal directions, the vertical and horizontal vibration signals were weak, and peak amplitudes were close to each other. Hence, the axial vibration signals were found to be more informative. The axial RMS vibration values of the training sets shown in Table 7 can be used only for the detection of MC 3. All other machine conditions have very close RMS values, which prevents the use of this feature for the detection of fault conditions of a centrifugal blower.

The maximum amplitudes of all vibration signals are almost equal, and the maximum amplitude feature cannot be utilized for the detection of faults. The vibration crest factors of the four training sets can be utilized only for the detection of MC 3 (see Table 8). All other machine conditions have very close crest factors, which prevents the use of this feature for the de-
Table 8. AE crest factors.

\begin{tabular}{|c|c|c|c|c|c|c|}
\hline & 1 & 2 & 3 & 4 & Min & Max \\
\hline MC 1 & 2.999 & 3.018 & 3.056 & 3.068 & 2.999 & 3.068 \\
MC 2 & 3.003 & 2.999 & 3.008 & 2.992 & 2.992 & 3.008 \\
MC 3 & 2.671 & 2.696 & 2.733 & 2.740 & 2.671 & 2.740 \\
MC 4 & 2.994 & 3.023 & 3.066 & 3.073 & 2.994 & 3.073 \\
MC 5 & 2.962 & 2.997 & 3.051 & 3.052 & 2.962 & 3.052 \\
\hline
\end{tabular}

Table 9. AE crest factors.

\begin{tabular}{|l|c|c|c|c|c|c|}
\hline & 1 & 2 & 3 & 4 & Min & Max \\
\hline MC 1 & 922,397 & 913,554 & 889,123 & 884,541 & 884,541 & 922,397 \\
MC 2 & 918,251 & 918,949 & 920,443 & 920,779 & 918,251 & 920,779 \\
MC 3 & $1,167,622$ & $1,144,503$ & $1,114,004$ & $1,107,512$ & $1,107,512$ & $1,167,622$ \\
MC 4 & 927,033 & 911,303 & 883,697 & 881,439 & 881,439 & 927,033 \\
MC 5 & 946,480 & 927,465 & 892,592 & 885,805 & 885,805 & 946,480 \\
\hline
\end{tabular}

Table 10. Segmented FFT vibration spectra.

\begin{tabular}{|c|c|c|c|c|c||}
\hline $\mathrm{S}$ & MC 1 & MC 2 & MC 3 & MC 4 & MC 5 \\
\hline $10 \mathrm{~Hz}$ & 1 & 1 & 1 & 1 & 1 \\
$110 \mathrm{~Hz}$ & 1 & 1 & 1 & 1 & 1 \\
$210 \mathrm{~Hz}$ & 1 & 1 & 1 & 1 & 1 \\
$310 \mathrm{~Hz}$ & 1 & 0 & 1 & 1 & 1 \\
$410 \mathrm{~Hz}$ & 1 & 0 & 1 & 1 & 1 \\
$510 \mathrm{~Hz}$ & 1 & 0 & 1 & 0 & 1 \\
$610 \mathrm{~Hz}$ & 1 & 1 & 1 & 1 & 0 \\
$710 \mathrm{~Hz}$ & 0 & 0 & 1 & 0 & 0 \\
$810 \mathrm{~Hz}$ & 1 & 1 & 1 & 1 & 1 \\
$910 \mathrm{~Hz}$ & 1 & 1 & 1 & 1 & 0 \\
$1010 \mathrm{~Hz}$ & 0 & 1 & 1 & 1 & 0 \\
$1110 \mathrm{~Hz}$ & 0 & 1 & 1 & 1 & 0 \\
$1210 \mathrm{~Hz}$ & 0 & 1 & 1 & 1 & 0 \\
$1310 \mathrm{~Hz}$ & 0 & 0 & 1 & 1 & 0 \\
$1410 \mathrm{~Hz}$ & 0 & 0 & 1 & 1 & 0 \\
$1510 \mathrm{~Hz}$ & 0 & 0 & 1 & 0 & 0 \\
$1610 \mathrm{~Hz}$ & 0 & 0 & 1 & 0 & 0 \\
$1710 \mathrm{~Hz}$ & 0 & 0 & 1 & 0 & 1 \\
$1810 \mathrm{~Hz}$ & 0 & 1 & 1 & 1 & 1 \\
$1910 \mathrm{~Hz}$ & 0 & 1 & 1 & 1 & 1 \\
\hline
\end{tabular}

tection of fault conditions of a centrifugal blower.

The calculated energy values of the training sets shown in Table 9 can be utilized only for the classification of MC 3 . All other machine conditions have intersected values, which prevents the use of this feature for the detection of machine faults.

Figure 7 illustrates the difference in the values of RMS, crest factor, and energy time domain vibration signal features. Based on the values shown in Fig. 7, the vibration time domain features can be effectively utilized to detect MC 3. The features of other machine faults interfered, however, and cannot be utilized for detecting other machine faults such as MC 1, MC 2, MC 4 and MC 5.

Table 10 shows the detectability of all machine conditions at different segment sizes. ${ }^{12}$ All machine conditions were detected at a Segment size (S) of $210 \mathrm{~Hz}$ and a confidence level of 4. At segment sizes of 10 and $110 \mathrm{~Hz}$, all machine conditions were successfully detected. The confidence levels were 46 and 7 , respectively.

\subsection{Process Information Technique}

The average pressure information was selected to be investigated as a major process information for centrifugal blowers. A pressure sensor was installed in the air outlet tube to record the operating pressure. The average pressure shown in Table 11 was calculated based on four consecutive reading for each machine condition. The analysis showed that this feature can only be utilized for the classification of MC 2 . 


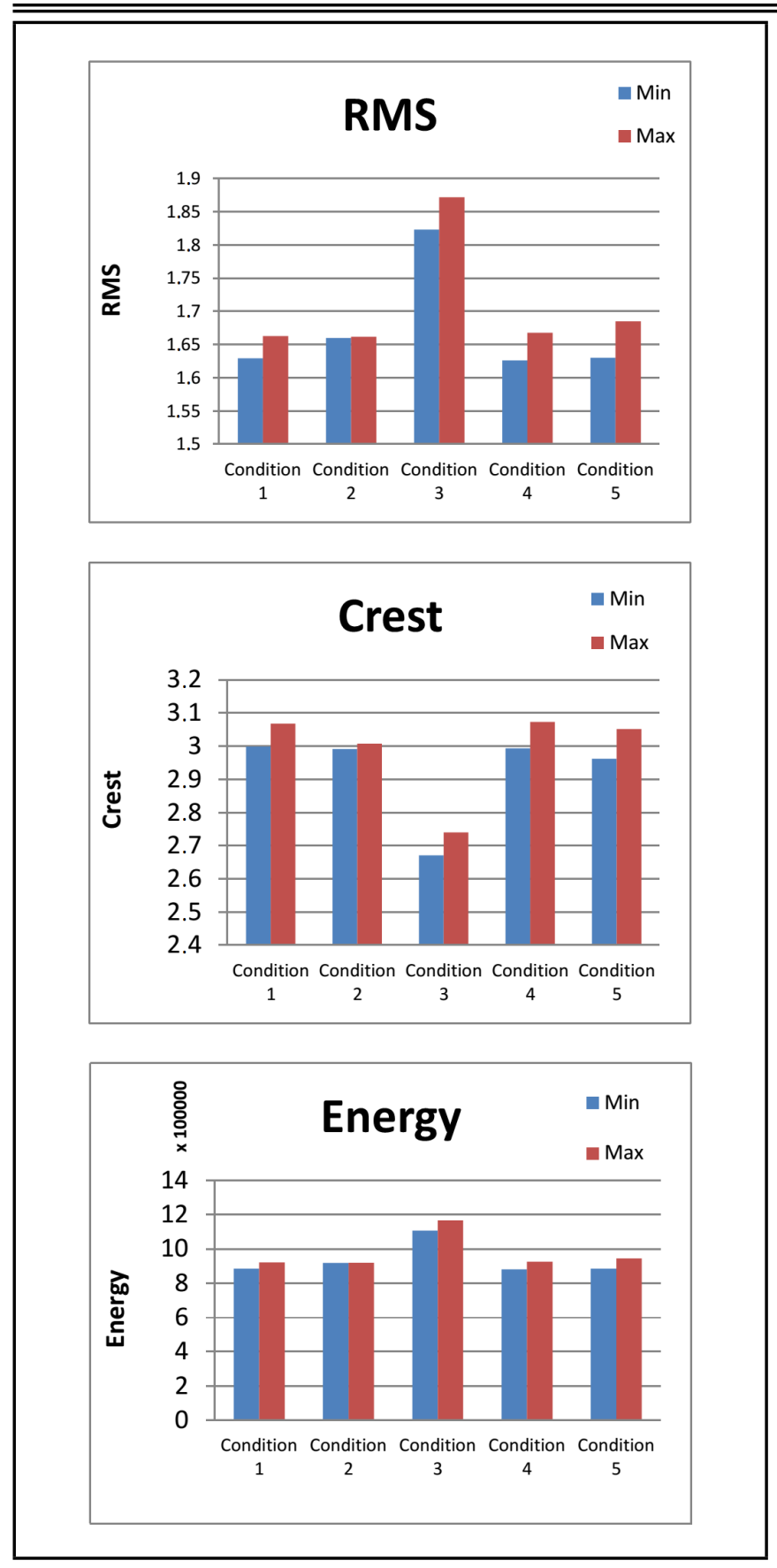

Figure 7. Graphical presentation for the vibration RMS, crest factor and energy features.

\section{RESULTS AND DISCUSSION}

A multi-fault detection technique was utilised for the condition-based monitoring of centrifugal blowers. Eleven features were extracted for each machine condition. The lookup table shown in Table 12 was built based on the results of benchmark thresholds and verification samples. "Weak" means that the minimum difference between benchmark threshold of this machine condition and the benchmark thresholds of other machine conditions (or the value confidence level) is less than or equal to 10 percent; "Good" means that the minimum difference is greater than 10 percent and less than 20 percent; "Strong" means that means that the minimum difference is greater than 20 percent but less than 30 percent; and "Very Strong" means that the minimum difference is equal to or greater than 30 percent. A tailor-made classification pro-
Table 11. Segmented FFT vibration spectra.

\begin{tabular}{|l|c|c|}
\hline & Average pressure ( BarA ) & Average pressure ( BarG ) \\
\hline MC 1 & 1.165 & 0.165 \\
MC 2 & 1.067 & 0.067 \\
MC 3 & 1.161 & 0.161 \\
MC 4 & 1.157 & 0.157 \\
MC 5 & 1.150 & 0.150 \\
\hline
\end{tabular}

gram was developed using MATLAB, based on the illustrated lookup table, and it yielded a detection accuracy of 100 percent. The use of multi-detection and multi-feature techniques significantly minimised the potential of fault interference and provided a better detection scheme.

Samples were collected from all machine conditions - 50 AE samples, 55 vibration samples, and 50 pressure samples. Of all the samples, 44 percent were utilised to identify the benchmark thresholds, and 56 percent were utilised for the evaluation of detection accuracy. RMS and energy features of AE signals proved their efficiency in detecting MC 1, MC 2, MC 4, and group 2 with a detection accuracy of 100 percent. The crest factor and amplitude features detected MC 4, group 1, and group 2 with a detection accuracy of 100 percent. The main drawback of the AE time domain features is that MC 3 and MC 5 are always undetectable. The AE spectral features proved their effectiveness over time domain features, as they successfully detected all faults at any segment size smaller than or equal to $108 \mathrm{kHz}$ with a detection accuracy of 100 percent.

RMS, amplitude, crest factor, and energy features of vibration signals demonstrated their efficiency in detecting MC 3 with a detection accuracy of 100 percent. The main drawback of the vibration time domain features is that MC 1, MC 2, MC 4, and MC 5 are undetectable. The vibration spectral features failed to detect all machine conditions at segment sizes of 200 and $300 \mathrm{~Hz}$. However, the vibration spectral features technique proved its greater effectiveness over time domain features, as it successfully detected all machine conditions at a segment size of $100 \mathrm{~Hz}$ with a detection accuracy of 100 percent. The failure in detecting faults at 110 and $210 \mathrm{~Hz}$ was expected due to the small confidence level values at those segment sizes. The confidence level values at 10, 110, and $210 \mathrm{~Hz}$ are 46,7 , and 4 , respectively. ${ }^{12}$

The pressure information proved its efficiency in detecting MC 2 with an accuracy of 100 percent. The main drawback of this technique is that the pressure information did not provide enough information for the detection of other machine conditions. It can be observed that fault interference occurred in MC 5, as the faults of MC 3 and MC 4 interacted together and produced a new fault signature. This study is limited to similar high-speed industrial centrifugal blowers, and was carried out at a specific ambient temperature and operation time. Due to the similarity between blowers and compressor, the results of this study can be extended to centrifugal compressors.

\section{CONCLUSION}

The presented work investigated bearing and air leakage faults of centrifugal blowers using three major fault detection techniques - namely acoustics, vibration, and pressure information. The proposed "lookup" table provides an integrated solution for the fault diagnostics of typical centrifugal equipment to maximise the accuracy of detection and to avoid false 
Table 12. A lookup table for the classification of centrifugal blower faults.

\begin{tabular}{|c|c|c|c|c|c|c|c|}
\hline & MC 1 & MC 2 & MC 3 & MC 4 & MC 5 & Group 1 & Group 2 \\
\hline $\begin{array}{c}\text { AE } \\
\text { RMS } \\
\text { Amplitude } \\
\text { CF } \\
\text { Energy } \\
\text { FFT @S }<90 \mathrm{kHz}\end{array}$ & $\begin{array}{c}\text { Weak } \\
\mathrm{X} \\
\mathrm{X} \\
\text { Weak } \\
\text { Very Strong }\end{array}$ & $\begin{array}{c}\text { Weak } \\
\mathrm{X} \\
\mathrm{X} \\
\text { Weak } \\
\text { Very Strong }\end{array}$ & Very Strong & $\begin{array}{c}\text { Very Strong } \\
\text { Very Strong } \\
\text { Weak } \\
\text { Very Strong } \\
\text { Very Strong }\end{array}$ & $\begin{array}{c}\mathrm{X} \\
\mathrm{X} \\
\mathrm{X} \\
\mathrm{X} \\
\text { Very Strong }\end{array}$ & $\begin{array}{c}\text { X } \\
\text { Strong } \\
\text { Weak } \\
\text { X } \\
\text { X }\end{array}$ & $\begin{array}{c}\text { Strong } \\
\text { Strong } \\
\text { Weak } \\
\text { Very Strong } \\
\text { X }\end{array}$ \\
\hline $\begin{array}{c}\text { Vibration } \\
\text { RMS } \\
\text { Amplitude } \\
\text { CF } \\
\text { Energy } \\
\text { FFT@ } \mathrm{S}=10 \mathrm{~Hz}\end{array}$ & $\begin{array}{c}X \\
X \\
X \\
X \\
\text { Good }\end{array}$ & $\begin{array}{c}\mathrm{X} \\
\mathrm{X} \\
\mathrm{X} \\
\mathrm{X} \\
\text { Good }\end{array}$ & $\begin{array}{l}\text { Good } \\
\text { X } \\
\text { Weak } \\
\text { Good } \\
\text { Good }\end{array}$ & $\begin{array}{c}X \\
X \\
X \\
X \\
\text { Good }\end{array}$ & $\begin{array}{c}X \\
X \\
X \\
X \\
\text { Good }\end{array}$ & $\begin{array}{l}X \\
X \\
X \\
X \\
X\end{array}$ & $\begin{array}{l}X \\
X \\
X \\
X \\
X\end{array}$ \\
\hline $\begin{array}{c}\text { Pressure } \\
\text { Average (BarG) }\end{array}$ & $\mathrm{X}$ & Very strong & $\mathrm{X}$ & $\mathrm{X}$ & $\mathrm{X}$ & $\mathrm{X}$ & $\mathrm{X}$ \\
\hline
\end{tabular}

alarms. An accurate assessment of the three major conditionbased monitoring techniques was given in this article using five time domain and frequency domain features, with a total number of 11 different feature sets for each machine condition.

$\mathrm{AE}$ and vibration time domain features failed to detect the 5 addressed machine conditions, while the $\mathrm{AE}$ and vibration frequency domain features managed to detect all of the addressed faults with a detection accuracy of 100 percent. The pressure information was only useful in detecting the air leakage problem (MC 2). The AE technique proved its greater effectiveness over vibration and pressure information techniques, except in the case of leakage, where the pressure information technique was competitive. In comparison to time domain features, the FFT spectral (frequency domain) features were best for the detection of high-speed centrifugal air blower faults. The fault interference occurred during experimentation. Two faults signals, MC 3 and MC 4, interacted with each other in an unexpected way, which resulted in new fault signatures. Fault interference usually results in a new fault signature, or in masking one or more of the existing failures. A full capacity CBM system that collectively use the best features and fault detection techniques can be developed based on the results of this study. The collective utilization of the major signal features and fault detection techniques could have the potential to reduce false alarms due to noise and fault interference issues.

Future research could investigate other blower faults in addition to the utilisation of different faults detection techniques, such as temperature measurements, thermal imaging, and wavelet analysis. Moreover, further experimentation could be carried out to apply the result of this study to similar industrial centrifugal blowers and compressors at different conditions or to different types of blowers. The issue of fault interference exists, and needs further investigation.

\section{REFERENCES}

1 Rienstra, A. Predictive Maintenance for the Masses, Airbone Ultrasonic, SDT North America, (2005).

2 Viktorov, I. Rayleigh and Lamb Waves, Physical Theory and Applications, Springer, USA, (2013).

3 Shepard, A. and Dennis, G. Principles of Turbomachinery, McMillan, USA, (1956).

4 Scheer, C., Südmersen, U., Reimche, W., and Bach, W. Quality control using vibration and acoustic signatures, $3 r d$
PAN American Conference for Non-Destructive Testing PANNDT, Rio de Janeiro, Brasil, (2003).

5 Implementation strategies and tools for condition based maintenance at nuclear power plants, IAEA-TECDOC1551, International Atomic Energy Agency, (2007).

6 Mathews, J. Acoustic Emission, Gordon and Breach Science Publishers Inc, USA, (1983), $3^{\text {rd }}$ edition.

7 Al-Ghamd, A. and Mba, D. Comparative experimental study on the use of Acoustic Emission and vibration analysis for bearing defect identification and estimation of defect size, Mechanical Systems and Signal Processing, 20 (7), 1537-1571, (2006).

8 Tandon, N. and Nakra, B. Defect detection of rolling element bearings by acoustic emission method, Journal of Acoustic Emission, 9 (1), 25-28, (1990).

9 Rogers, L. The application of vibration analysis and acoustic emission source location to on-line condition monitoring of anti-friction bearings, Journal of Tribology International, 12 (2), 51-58, (1979).

10 Wang, J. and $\mathrm{Hu}, \mathrm{H}$. Vibration-based fault diagnostic of pump using fuzzy technique, Measurement, 39 (2), 176185, (2006).

11 Schulthesis, S., Lickteig, C., and Parchewsky, R. Reciprocating compressor condition monitoring, Proceedings of the $36^{\text {th }}$ Turbo Machinery Symposium, Texas, USA, (2007).

12 Gowid, S., Dixon, R., and Ghani, S. FFT-based robust automated segmentation and features selection algorithm for condition based monitoring systems, Applied Acoustics, 88 (1), 66-74, (2015).

13 Barden Precision Bearings, Bearing failure: causes and cures, Retrieved from http://www.schaeffler.com /remotemedien/media/_shared_media/08_media_library /01_publications/barden/brochure_2/downloads_24 /barden_bearing_failures_us_en.pdf, (Accessed March 10, 2014).

14 Gowid, S., Dixon, R., and Ghani, S. Profitability, reliability and condition based monitoring of LNG floating platforms: A review, Journal of Natural Gas Science and Engineering (Elsevier), 27 (3), 1495-1511, (2015). http://dx.doi.org/10.1016/j.jngse.2015.10.015 